page and the second study-trial page. All Ss were told that their task would be to memorize a list of 24 nouns. They were given $48 \mathrm{sec}$ to study the nouns and $3 \mathrm{~min}$ to recall them. The $S s$ in the sentence-context and scrambled-sentence context conditions were told that there would be other words on the study page in addition to the underlined nouns. They were assured that they would not be asked to recall the additional words. The conceptually related words were identical to the ones used by Cofer in his first experiment. The "unrelated" nouns were selected randomly from the Thomdike-Lorge (1944) norms, with the restriction that the words were high-frequency nouns ( $A$ and above). The words were separated into six groups of four each so that a sentence could be constructed that used all four words. It is probable that some of the nouns in the "unrelated" list were related, but the list was "unrelated" in comparison to the list composed of six nouns from each of four categories. Seven Ss in each condition received the related list first, and eight $S s$ received the unrelated list first. A total of 45 students from undergraduate psychology courses at Michigan State University were assigned randomly to the three conditions so that there were $15 \mathrm{Ss}$ in each condition.

\section{Results and Discussion}

The mean number of words correctly recalled for the sentence, scrambled-sentence, and control conditions was $14.33,14.53$, and 14.40 , respectively, for the "unrelated" list of nouns, and $14.87,15.67$, and 17.80 , respectively, for the related list of nouns. An analysis of variance revealed that the only significant effect was due to the nature of the lists, $\mathrm{F}=4.82, \mathrm{df}=1 / 42, \mathrm{p}<.05$. If only the related words are considered, as a check of the reliability of the findings of Experiment 1, the orthogonal comparison between the sentence and control conditions yields a significant effect, $F=5.80, \mathrm{df}=1 / 42, \mathrm{p}<.05$. The mean ratio of obtained clusters to maximum possible clusters, based on concept categories, was $59 \%, 69 \%$, and $83 \%$ for the sentence, scrambled-sentence, and control Ss, respectively. The only significant effect was the orthogonal comparison between the sentence and control conditions, $\mathrm{F}=4.41, \mathrm{df}=1 / 42, \mathrm{p}<.05$. Thus, once again, the presence of sentences as context had a deleterious effect on the recall and clustering of related nouns. Yet, the failure to find much of a difference between the sentence context and scrambled-sentence context conditions does not provide much support for the view that the ordering of the context words as sentences is the important factor. Moreover, if the ordering of the context words was important, the cluster scores based on the sentences (i.e., the four nouns in each sentence would be a category) should have revealed greater clustering for the sentence condition than for the scrambled-sentence condition. However, this was not the case since the mean ratio of obtained clusters to maximum possible clusters for the related list was $22 \%$ and $24 \%$ for the sentence and scrambled-sentence conditions, respectively.

In conclusion, the present study extends Cofer's finding that sentence context has a deleterious effect on the recall of nouns when conceptually related nouns are presented in different sentences. The context effect was obtained even when the method of complete presentation was used and the nouns to be recalled were underlined. There was weak evidence that the sentence context effect was due to the presence of additional words and not to the ordering of the additional words as sentences.

REFERENCES

COFER, C. N. Free recall of nouns after presentation in sentences. Journal of Experimental Psychology, 1968, 78, 145-152. THORNDIKE, E. L., \& LORGE, I. The teacher's word book of 30,000 words. New York: Bureau of Publications, Teachers College, Columbia University, 1944.

$$
\text { NOTE }
$$

1. This investigation was supported by Grant MH 16991 from the National Institute of Mental Health and by a Biomedical Sciences Support grant from Michigan State University. Thanks are due to Joyce Pennington for assistance during all phases of the investigation.

\title{
Short-term memory: Effects of type of trigram isolated and position of isolation
}

\section{RICHARD S. CIMBALO and MARGARET S. MAHONEY, Rosary Hill College, 4380 Main St., Buffalo, N.Y. 14226}

The effects of five different types of trigram units, embedded in three positions (beginning, middle and end) of an eight-item list of consonants, were investigated. The five types of trigrams were: letter repetitions (GGG), meaningful-pronounceable (FIB), meaningful-unpronounceable (FBI), unmeaningful-pronounceable $(B I F)$, and unmeaningful-unpronounceable (BGF). Voice differences were used to achieve isolation, and a free recall procedure was employed. The data indicated an inverse relationship between both overall list performance and isolated trigram performance and the other items in the list.

This study was designed to investigate the effect of varying the type of isolated unit upon recall performance for the unit and performance on the other items in a serial list. If a meaningful-pronounceable (MP) trigram were somehow made more perceptually distinct in a list of consonants, would the performance for that unit and for the other items in the list differ from performance on a list where an unmeaningful-unpronounceable (UU) trigram occurred as a distinct item in a list? The MP item would be easier to encode and/or rehearse (Laughery \& Pinkus, 1968), and it seems tenable to assume that performance for the MP trigram and for the unisolated items in such a list to be superior when compared to the UU condition. However, besides the ease of the processes of encoding and rehearsal, the attention-getting value of the stimulus and its effects upon the S's strategy must be taken into consideration (viz, Gregg \& Simon, 1967). The MP condition may utilize more of the S's processing time because of its structural distinctiveness (i.e., vividness in a list).

\section{METHOD}

The Ss were 50 female undergraduate students from Rosary Hill College who volunteered their time and service. A 3 by 5 factorial design was employed. Position of the chunk was a within-S variable with three levels: isolated unit at the beginning of a list (i.e., Positions 1,2 , and 3), middle (i.e., Positions 5, 6, and 7), or end (i.e., Positions 9,10, and 11). The type of chunk was a between-S variable consisting of five levels: letter repetitions (LR), meaningful-pronounceable (MP), and meaningful-unpronounceable (MU), unmeaningful-pronounceable (UP), and unmeaningful-un pronounceable (UU). Examples of each of the preceding types of chunks are: GGG, FIB, FBI, BIF, and BGF, respectively.

Each $\mathrm{S}$ was given 1811 -item lists (i.e., 6 lists with the isolated unit at each of the three positions). The position variable was randomized within a set of 18 lists, and this same random order was used for all five type-of-chunk conditions. Eight letters of each list were generated randomly from the 20 consonants of the alphabet ( $Y$ was considered a vowel) without replacement.

The MU, MP, and UP units were taken from a recent study by Laughery \& Pinkus (1968). The LR units were consonants chosen at random. The UU chunks were randomized consonants. 
The isolation effect of each condition was achieved by having a female voice record the eight randomized consonants and a male voice record the chunks. Since the male and female voices occurred the same number of times in each condition and since all the comparisons, are within-voice ones, counterbalancing of voice was deemed necessary.

All items (letters) were recorded for presentation on a Viking Model 880 tape recorder. The items were recorded at a 60 items $/ \mathrm{min}$ rate, with a 20 -sec interval in which Ss could report their responses.

Ten Ss were tested per condition. They were instructed as to the construction of the list (i.e., 11 items/list, type of chunk, male and female voice). Each group was given identical instructions except for the description of the type of chunk that would be presented. The Ss were told to record their answers when the word "record" was heard. The record signal occurred $1 \mathrm{sec}$ after the last item. A free-recall procedure was used (i.e., the Ss could record the items in any order they chose).

\section{RESULTS}

An analysis of variance was performed on the total number of correct items (letters), including the items in the isolated unit. Type of chunk was found to be significant, with $F(4,45)=9.30, p<.01$. The results are plotted in Fig. 1 (solid bar). As can be seen in the figure, overall performance becomes progressively worse for Conditions LR, MP, MU, UP, and UU, respectively. Overall performance was found to be best for letter repetitions. Also, the overall performance was superior for meaningful conditions at both levels of pronunciability. Meaningfulness seems to benefit overall performance more than pronunciability. A significant Type of Chunk by Position of Chunk interaction $[F(8,90)=3.95, p<.01]$ indicated that Conditions MU, UP, and UU display

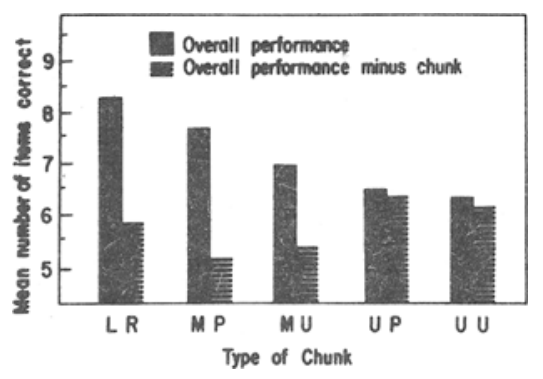

Fig. 1. Mean number of items correct per sequence and the mean number of items correct per sequence excluding the isolated unit for the five types of isolated units. greater differences when the isolated item occurs at the beginning of the list, and these differences are minimal when the isolated item occurs at the end of the list.

An analysis was performed for the number of other items correct (disregarding the isolated unit) in the list. Again, the type of chunk was found to be significant, with $\mathrm{F}(4,45)=6.95, \mathrm{p}<.01$. The data appears in Fig. 1 (lined bar). The trend for performance on the other items in the list was the reverse of the overall analysis. Performance on the other items in the list was superior for unmeaningful conditions at both levels of pronunciability. Meaningfulness of a chunk seems to retard performance on the other items in the list relative to the effect of unmeaningful chunks. Letter repetitions exert an effect on other items in the list intermediate to the effects exerted by meaningfulness and pronunciability. A significant Type of Chunk by Position of Chunk interaction $[F(8,90)=4.60$, $p<.01]$ indicated that differences among the various types of chunk conditions were very small when the chunks occurred in the beginning of the list but that differences did occur for the middle and end positions. In general, when the chunk occurred in the middle and end of the list, the unmeaningful chunks (UU and UP) yielded better performance on the other items in the list than did the meaningful or letter repetition chunks.

A third analysis was performed on the total number of chunks correct. In this analysis, type of chunk was found to be significant, with $F(4,45)=38.74, p<.001$. The results paralleled the overall item performance data (Fig. 1, clear bar).

\section{DISCUSSION}

The results indicate that overall performance and performance on the isolated trigram are inversely related to performance on the other items in the list. The better the performance on the isolated item, the worse the performance for the other items in the list. The more meaningful a chunk and the easier it is to pronounce (in that order), the better is performance on that item but at the expense of performance on the other items in the list.

Gibson et al (1964) have shown that, in a tachistoscopic-threshold experiment, UP units have a lower recognition threshold than do MU units. This indicates that pronunciability is a more effective encoding characteristic than is meaningfulness. Gibson et al, in the same article, also reported that free-recall performance for a list of trigrams produced significantly better performance for the MU units. Hence, meaningfulness would appear to be a more effective characteristic for rehearsal processes. The plot of the interaction of the overall analysis indicated that the condition MU depicted a very sharp decline as the isolated unit approached the end of the list, until in the ultimate position, it did not differ from Conditions UP and UU. This effect seems to also infer that meaningful (MU) units were remembered better than were pronounceable (UP) units, not because they were easier to encode, but rather because they were easier to rehearse once they were encoded.

As regards the other items in the list (i.e., excluding the isolated unit), the performance was the inverse of that found for overall performance. The interaction found in this analysis indicates that the benefit derived by the other items is a function of where in the list the isolated unit occurs as well as the type of trigram. Why meaning should have a more deleterious effect on the other items in the list when this characteristic occurs in the middle and end positions is not entirely clear.

An interference theory would have some difficulty trying to explain the inverse relationship between the performance measures. Reduced intralist interference would seem to predict opposite results from those found here. An information-processing view which concerned itself only with ease of encoding and/or rehearsal for the isolated item would make predictions similar to an interference theory. A theory that would seem to be better able to handle these results would be one that stressed S's strategies in processing information in addition to memory structure (Miller, Galanter, \& Pribram, 1960; Atkinson \& Shiffrin, 1969).

\section{REFERENCES}

ATKINSON, R. C. \& SHIFFRIN, R. M. Human memory: A proposed system and its control processes. In K. W. Spence and J. T. Spence (Eds.), The psychology of learning and motivation: Advances in research and theory. Vol. II. New York: Academic Press, 1969.

GIBSON, E. J., BISHOP, C. H., SCHIFF, W., \& SMITH, J. Comparison of meaningfulness and pronunciability as grouping principles in the perception and retention of verbal material. Journal of Experimental Psychology, 1964, 67, 173-182.

GREGG, L. W., \& SIMON, H. A. An information-processing explanation of one-trial and incremental learning. Journal of Verbal Learning \& Verbal Behavior, 1967, 6, 780-787.

LAUGHERY, K. R., \& PINKUS, A. L. Recoding and presentation rate in short-term memory. Journal of Experimental Psychology, 1968, 76, 636-641.

MILLER, G. A., GALANTER, E., \& PRIBRAM, $\mathrm{K}$. H, Plans and the structure of behavior. New York: Holt, Rinehart \& Winston, 1960. 